\title{
Contribuição de acadêmicos de enfermagem na melhoria da qualidade de vida de idosos institucionalizados: revisão
}

RESUMO | Objetivo: Visou realizar a identificação das ações que quando executadas por acadêmicos de enfermagem propiciam uma melhoria da qualidade de vida de idosos institucionalizados. Método: Trata-se de uma revisão integrativa construída a partir da questão norteadora "Quais as contribuições dos acadêmicos de enfermagem para melhoria da qualidade de vida dos idosos institucionalizados?" Após o processo de busca na literatura foram selecionados 9(100\%) artigos, sendo o ano de 2016 o de maior frequência de publicações com 3(33,33\%) artigos encontrados. Resultados: Diante da leitura e estudo da literatura constatou-se que as atividades desenvolvidas pelos discentes juntamente os idosos, surtiram efeitos positivos, os quais podem colaborar para a manutenção da saúde e interação social. Conclusão: Com os resultados obtidos acredita-se que, ao pôr em prática esses métodos, oferta-se uma forma de envelhecimento saudável e ativo. Isso traz em tona a discussão sobre os fatores que influenciam na qualidade de vida dos idosos institucionalizados.

Palavras-chaves: Instituição de Longa Permanência para Idosos; Qualidade de vida; Estudantes de enfermagem.

\begin{abstract}
Objective: To identify actions that, when nursing students perform, provide an improvement in the quality of life of institutionalized elderly. Method: This is an integrative review constructed from the issue of guidance "What are the contributions of nursing students to improve the quality of life of institutionalized elderly?" after the literature search process, 9 (100\%) 2016 as the most frequent number of publications with $3(33.33 \%)$ found items. Results: In view of reading and studying the literature, it was found that the activities developed by the students together with the elderly had positive effects, which can contribute to the maintenance of health and social interaction. Conclusion: With the results obtained it is believed that, putting these methods into practice, a healthy and active form of aging is offered. This raises the debate about the factors that influence the quality of life of institutionalized elderly. Keywords: Homes for the Aged; quality of life; Students.
\end{abstract}

RESUMEN | Objetivo: Identificar las acciones que, al realizar los estudiantes de enfermería, proporcionen una mejora en la calidad de vida de los ancianos institucionalizados. Método: Se trata de una revisión integradora construida a partir de la cuestión de la orientación "¿Cuáles son las contribuciones de los estudiantes de enfermería para mejorar la calidad de vida de los ancianos institucionalizados?", después del proceso de búsqueda de literatura, 9 (100\%) 2016 como el número más frecuente de publicaciones con $3(33,33 \%)$ artículos encontrados. Resultados: En vista de la lectura y el estudio de la literatura, se encontró que las actividades desarrolladas por los estudiantes junto con los ancianos tuvieron efectos positivos, que pueden contribuir al mantenimiento de la salud y la interacción social. Conclusión: Con los resultados obtenidos se cree que, poniendo en práctica estos métodos, se ofrece una forma saludable y activa de envejecimiento. Esto plantea el debate sobre los factores que influyen en la calidad de vida de los ancianos institucionalizados. Palavras claves: Hogares para Ancianos; calidad de vida; Estudiantes de Enfermería.

\section{Thays Cristina Pereira Barbosa}

Graduada em Enfermagem, Universidade do Estado de Minas Gerais, Minas Gerais, Brasil. ORCID: 0000-0002-9352-0907

\section{Allan de Morais Bessa}

Graduando em Enfermagem, Universidade do Estado de Minas Gerais, Minas Gerais, Brasil. ORCID: 0000-0001-6969-7537

\section{Fernanda Oliveira de Assis}

Graduada em Enfermagem, Universidade do Estado de Minas Gerais, Minas Gerais, Brasil. ORCID: 0000-0002-4339-6274

\section{Fernanda Marcelino de Rezende e Silva}

Mestre em Enfermagem pela UFMG, Docente do Curso de Graduação em Enfermagem da Universidade do Estado de Minas Gerais (UEMG), Unidade Divinópolis. ORCID: 0000-0003-2236-7009

Recebido em: 17/07/2020

Aprovado em: 24/08/2020

\section{Regina Consolação dos Santos}

Enfermeira. Mestre em Ciência pela Universidade Federal de São João Del Rei, Campus Centro Oeste Dona Lindu UFS (CCO). Docente do Curso de Graduação em Enfermagem da Universidade do Estado de Minas Gerais (UEMG), Unidade Divinópolis. ORCID: 0000-0002-7393-3210

\section{Silmara Nunes Andrade}

Enfermeira. Doutora em Ciências da Saúde pela Universidade Federal de São João Del Rei, Campus Centro Oeste Dona Lindu UFSJ (CCO). Docente do Curso de Graduação em Enfermagem da Universidade do Estado de Minas Gerais (UEMG), Unidade Divinópolis. ORCID: 0000-0002-1975-0827
INTRODUÇÃO

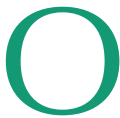
envelhecimento populacional constitui um dos maiores desafios da saúde pública contemporânea, onde o aumento da população idosa no mundo tem representado uma grande mudança na sociedade ${ }^{1}$. O processo de envelhecimento caracteriza-se como um processo progressivo e irreversível, ligado aos fatores biológicos, sociais e psicológicos ${ }^{2}$. Contudo, esse processo é individual, não sendo somente devido a condição genética e sim dos hábitos que temos ao longo da vida. O enveIhecimento é um processo natural que se evidencia com o tempo, entretanto, esse processo vai depender do histórico de vida aliado as potencialidades genéticas ${ }^{3}$.

De acordo com a Organização das Nações Unidas- ONU4, em 1950, apenas 
8\% da população mundial estava com 60 anos ou mais. Em 2013, essa proporção subiu para $12 \%$ e espera-se que atinja 21\%, passando de 841 milhões em 2013 para mais de 2 bilhões de idosos em 2050. Desde 2012, houve um aumento significativo desse grupo populacional, superando a marca dos 30,2 milhões de idosos em $2017^{5}$. São considerados idosos os indivíduos com 65 anos ou mais, no entanto, em países em desenvolvimento, porém, devido à baixa expectativa de vida, o limite de idade é de 60 anos $^{6}$.

Dentre os fatores relacionados ao envelhecimento populacional, pode ser destacado algumas mudanças de indicadores de saúde como: a diminuição da fecundidade e principalmente da mortalidade, levando em peso o então aumento da expectativa de vida ${ }^{7}$. O avanço tecnológico na área da saúde é um dos mecanismos que explica o então aumento no tempo de vida, pois com a redução da letalidade de algumas doenças, houve um aumento de morbimortalidades, sendo assim, pessoas vivendo com doenças crônicas ${ }^{8,9}$.

Com a progressão do processo de envelhecimento, a qualidade de vida (QV) do indivíduo é fortemente determinada pela habilidade de manter sua independência e, autonomia ${ }^{10}$. Visto isso, faz-se necessário a realização do levantamento científico sobre o processo de envelhecimento e a qualidade de vida ${ }^{11}$. A definição de QV é entendido enquanto a percepção do indivíduo sobre sua posição na vida, sendo contextualizado pela cultura e levando em consideração o sistema de valores vividos, e em relação aos objetivos, expectativa, padrões e preocupações ${ }^{11}$.

Diante disto, é possível observar que quando se trata do processo de envelhecimento a QV geralmente é relacionada a manutenção da autonomia, essa que pode ser vista em atividade diárias feitas por idosos com capacidade funcional ${ }^{12}$. A autonomia e a capacidade funcional estão diretamente ligadas à obtenção da QV, e ao envelhecimento saudável. Assim, a equipe multiprofissional deve promover atividades direcionadas à ma- nutenção da funcionalidade do idoso, com o intuito de prevenir ou protelar a incapacidade, limitações e dependência da pessoa idosa ${ }^{10}$.

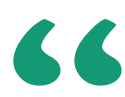

As Instituições de Longa Permanência para Idosos (ILPI's) têm como objetivo garantir a atenção integral às pessoas com mais de 60 anos, defendendo a sua dignidade os seus direitos. São instituições que buscam prevenir a redução dos riscos aos quais ficam expostos os idosos que não contam com uma moradia ${ }^{13}$. Apesar das ILPI's atenderem às necessidades do idoso, como moradia, alimentação, boa higiene e acompanhamento médico, há uma espécie de isolamento de suas atividades familiares e sociais, que devido a certas limitações as ILPI's nem sempre conseguem suprir todas as necessidades biopsicossociais de seus residentes afetando diretamente a QV desta população ${ }^{10}$.

O cuidado a idosos institucionalizados vem preocupando a sociedade devido ao crescente aumento da população idosa no Brasil. Em geral, o perfil do idoso institucionalizado caracteriza-se pelo aumento do sedentarismo, a perda da autonomia e da autoestima, a ausência de familiares, além das influências de fatores biológicos, doenças e outras causas externas comuns a essa fase de envelhecimento, destacando a ocorrência de quedas como um dos agravos mais importante à saúde ${ }^{14}$.

Nesse contexto, destaca-se a Extensão Universitária, por configurar nova estratégia que favorece o processo de ensino-aprendizagem, o qual articula saberes que envolvem o meio acadêmico, científico e a comunidade, colaborando para a formação de cidadãos com novas formas de pensar e agir na saúde. Além disso, oportuniza o saber recíproco, com ênfase em uma comunicação dialógica, favorecendo conhecer as necessidades e potencialidades dos indivíduos, das famílias, induzindo a participação efetiva dos envolvidos ${ }^{15,16}$.

No tocante aos acadêmicos das diversas áreas da saúde, estes devem atuar privilegiando o diálogo, respeitando a individualidade de cada pessoa, conduzindo o processo educativo de modo em que os indivíduos não sejam apenas ouvintes passivos, mas sim, críticos e reflexivos ${ }^{17}$.

Vale destacar que, na esfera da saúde, 
o papel da extensão universitária visa promover, por meio de qualquer recurso, uma melhora da qualidade de vida de uma comunidade alvo, inserida em uma população de risco. Ademais, em relação ao aspecto acadêmico, cria-se a oportunidade dos discentes em Enfermagem relacionarem o conteúdo teórico com a realidade da prática, contribuindo para o desenvolvimento de trabalhos extracurriculares que complementem o aprendizado e aprimorem as habilidades sociais necessárias para a profissão, além de possibilitar aos acadêmicos um aprendizado prático, ao mesmo tempo em que prestam serviços à comunidade atendida ${ }^{18,19}$.

Assim, este estudo teve como objetivo identificar as ações dos acadêmicos de enfermagem para melhoria da qualidade de vida dos idosos institucionalizados.

\section{MÉTODO}

A revisão integrativa consiste no cumprimento das etapas: identificação do tema e seleção da questão de pesquisa; estabelecimento dos critérios de elegibilidade; identificação dos estu-

FIGURA 1 - Resultado quantitativo referente à busca nas bases de dados. Dos 9 artigos encontrados, 0 ano de $\mathbf{2 0 1 6}$ foi o de maior frequência de publicações com $3(33,33 \%)$ artigos.

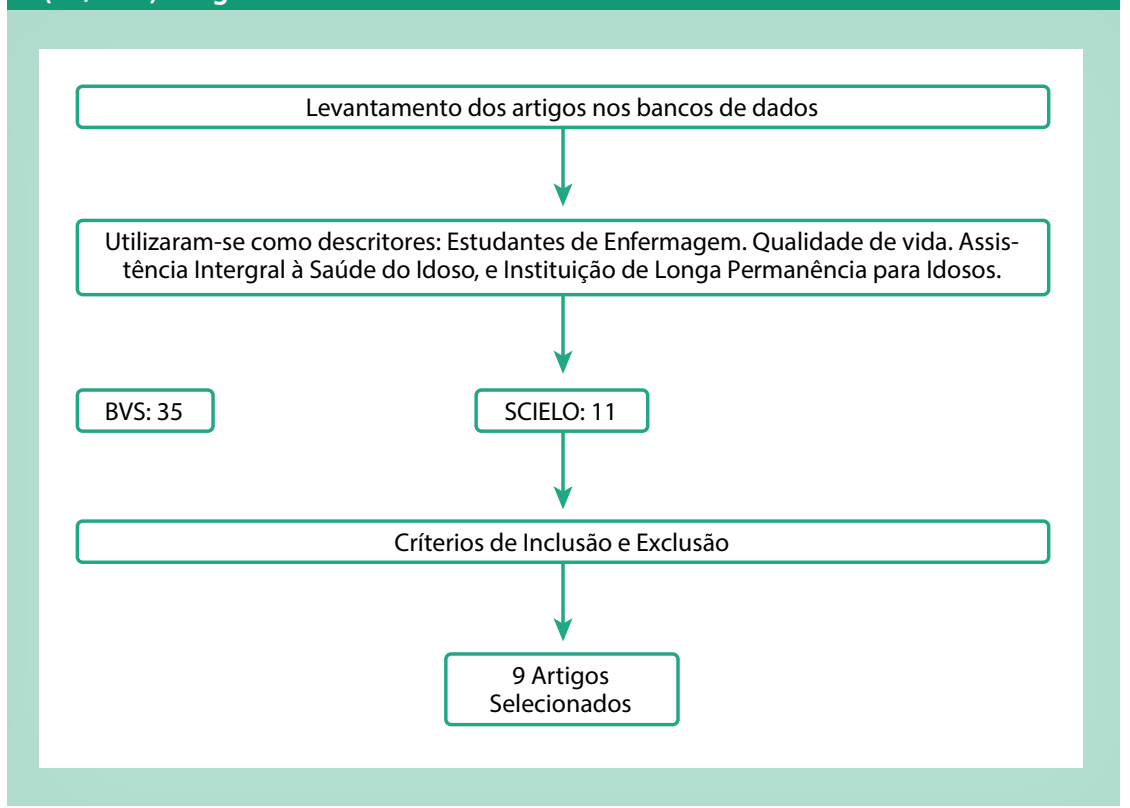

dos nas bases científicas; avaliação dos estudos selecionados e análise crítica; categorização dos estudos; avaliação e interpretação dos resultados e apresentação dos dados na estrutura da revisão integrativa $^{20}$. Sendo assim, a questão norteadora escolhida para construção do trabalho foi: "Quais as contribuições dos acadêmicos de enfermagem para melhoria da qualidade de vida dos idosos institucionalizados?".

Quanto ao levantamento bibliográfico foram consultadas as seguintes bases de dados: Scientific Electronic Library Online (SciELO), Medical Literature Analysis and Retrieval System Online (MEDLINE), Biblioteca Virtual em Saúde (BVS) e Ministério da Saúde. Utilizaram-se como descritores: Estudantes de enfermagem; Qualidade de vida; Assistência Integral à Saúde do Idoso; e Instituição de Longa de Permanência para Idosos. No cruzamento dos descritores foram adotados os operadores booleanos "AND" e "OR".

O planejamento de busca incluiu as seguintes ligações de palavras: Enfermeiras Estudantes OR Alunos de Enfermagem OR Estudante de Enfermagem OR Enfer- meiros Estudantes AND Qualidade de vida AND Assistência Integral à Saúde do Idoso OR Atenção Integral à Saúde do Idoso OR Atenção Integral ao Idoso OR Atendimento Integral à Saúde do Idoso AND Instituição de Longa Permanência para Idosos OR Asilo para Idosos OR Asilos para Idosos OR Ancianatos OR Casas de Repouso para Idosos OR Instituições Geriátricas de Longa Permanência OR Instituição Asilar OR ILPI.

Definiram-se os seguintes critérios de inclusão: artigos publicados na íntegra, disponíveis eletronicamente, em português, inglês e/ou espanhol, cujos resultados abordassem aspectos relacionados diretamente sobre a influência e benefícios das ações dos discentes de enfermagem que efetivamente contribuem na QV dos idosos em ILPI's. Foram excluídos os editoriais, cartas ao editor, dissertações, teses, revisões bibliográficas, e artigos com ano de publicação inferior à 2013. Portanto, no final da pesquisa, foi realizada uma leitura interpretativa dos teóricos, buscando analisar de forma mais ampla os conhecimentos.

Todos os artigos encontrados foram analisados e selecionados por dois pesquisadores da área de enfermagem. Cada observador verificou e complementou as informações obtidas individualmente e, por fim todas as divergências foram reavaliadas para aperfeiçoamento do estudo.

\section{RESULTADOS}

A coleta de dados consistiu na busca avançada nas bases de dados. Após o processo de seleção e identificação dos artigos que obedeceram aos critérios de inclusão estabelecidos: prévia leitura de todos os títulos, resumos ou abstract. Procedeu-se a leitura, sendo excluídos artigos não disponíveis na integra gratuitamente, que não contemplavam o objetivo proposto sendo seu ano de publicação inferior a 2013. Por fim, para realização do levantamento bibliográfico foram identificados nove artigos que abordam sobre QV no contexto da saúde da pessoa idosa. 
TABELA 1 - Distribuição dos artigos utilizados conforme detalhamento quantitativo, autores/ revista, título, objetivo, ano, 2013 - 2017.

$\mathbf{N}^{\circ} \quad$ Autores / Revista Título

Objetivo

Ano

Lucena et al.

01 Rev. Pesqui. Cuid.

Ensinando e aprendendo com idosos: relato de experiência.

Descrever um relato de práticas de educação em saúde de um projeto de extensão universitária, incentivando a adoção de medidas Fundam. preventivas de autocuidado em relação à saúde do idoso.

BALLA; SCORTEGAGNA.

02 Rev. Estudo interdisciplinar envelhecimento, Porto Alegre.

Uso do tempo livre através de recursos expressivos: Contribuição para um grupo de idosos institucionalizados.

BEZERRA et al.

03 Rev. Enferm. UFPE online.
Idosos institucionalizados: proposta educativa na perspectiva freireana.
Avaliar a contribuição do uso de recursos expressivos no tempo livre para mudanças no modo de ser e estar dos idosos residentes de uma ILP.

Refletir sobre as potencialidades e limitações das práticas de educação em saúde voltadas para os idosos residentes em instituições de longa permanência e apresentar uma proposta educativa que considere e respeite seus saberes e valores culturais.

FELIX et al.

04 Rev. Enferm. UFPE online. SILVA et al.

05 Revista de ciências da saúde.

Cuidados de enfermagem ao idoso

na instituição de longa permanência: relato de experiência.
Ações educativas vivenciadas com idosos: um relato de experiência.
Relatar a experiência de estudantes de enfermagem durante 0 período de estágio, observando a assistência de enfermagem aos idosos institucionalizados.

Relatar a experiência de acadêmicos da área de saúde, acerca das ações educativas mediante vivência com idosos no ano de 2016.

Relatar a experiência do Grupo do Programa de Educação Tutorial CYRINO et al.

06 Revista ciência em Extensão.

Atividades lúdicas como estratégia de educação em saúde com idosos.

Enfermagem da Universidade Federal de Goiás - Regional Jataí, na utilização de atividades lúdicas como estratégia de educação em saúde e integração social com idosos.

FLEURÍ et al.
07 Rev. Enfermagem
Revista. $\quad \begin{gathered}\text { Atividades lúdicas com idosos institu- } \\ \text { cionalizados }\end{gathered}$
REME - Ver. Min. Enferm

Oficina bingo da saúde: uma experiência de educação em saúde com Grupos de idosos.
0 intuito da proposta é proporcionar uma interação entre os institucionalizados, contribuindo de forma direta para uma melhor qualidade de vida.

Este estudo tem como objetivo relatar a experiência de integrantes do Programa de Educação Tutorial do curso de Enfermagem no projeto de extensão "Acampavida", realizado anualmente com idosos, por meio de atividades
CABRAL et al. Rev. Enfermagem 09

Promoção da Saúde.

Oficinas de educação em saúde com idosos: uma estratégia de Promoção da qualidade de vida
Realizar ações de Educação em Saúde voltadas para a Promoção da Saúde do idoso no Núcleo de Atenção ao Idoso da Universidade Federal de Pernambuco.

TABELA 2 - Eixos temáticos e número de atividades realizadas no período de 2013-2018.

\section{Eixos Temáticos}

Lazer

Educação em Saúde

Oficina Terapêutica

Atividades Neuromotoras

Cuidados de Enfermagem

As atividades realizadas pelos acadêmicos foram classificadas e ordenadas em 05 eixos norteadores que permitiram a discussão da temática, sendo respectivamente: "Lazer"; "Educação em saúde";
$\mathrm{N}^{\circ}$ de atividades

12

06

05

04

02

"Oficina terapêutica"; "Atividades neuromotoras"; e "Cuidados de enfermagem".

A tabela 2 demonstra os eixos temáticos e a quantidade de atividades realizadas pelos acadêmicos de enfermagem para a melhoria da qualidade de vida de idosos institucionalizados. Dos artigos pesquisados, a temática "educação em saúde" foi a de maior ocorrência com 5 (55,55\%) publicações.

\section{DISCUSSÃO}

As atividades lúdicas e o incentivo a momentos de lazer e recreação são formas eficazes para melhorar a autoestima, a qualidade de vida, o resgate da memória e estimular as funções cognitivas dos idosos. Buscando a melhor na qualidade de vida 
dos idosos, as atividades buscam uma meIhor adaptação dos pacientes a esse novo estilo de vida. São atividades simples e pouco utilizadas, que estão possibilitando novas e contínuas aprendizagens, além de relacionamentos sociais com manutenção da capacidade funcional ${ }^{21}$.

Por conseguinte, a recreação e o lazer necessitam ser potencializados em todo o percurso da vida, especificamente na terceira idade, quando os indivíduos se apresentam mais retraídos, afastados dos familiares e amigos, o que prejudica a realização de suas atividades de vida diária e dificulta sua convivência no contexto familiar e social ${ }^{21,22}$.

Em relação as ações educativas, estas têm como objetivo, promover a saúde, ações preventivas, interação social e o autocuidado. Visam também, a promoção, a proteção e, principalmente, a prevenção de agravos. Além disso, estimula as pessoas a refletirem sobre sua condição de vida, saúde e as ações exercidas em relação ao autocuidado, como também, sua interação entre condição de saúde, o meio o qual está inserido e o contexto familiar ${ }^{23}$.

Essas atividades educativas colaboram para a manutenção da saúde dos idosos, edifica mudanças no cotidiano, favorece a reflexão entre o saber popular e o científico, proporciona novos saberes que influenciam as atitudes e práticas, motivando o desenvolvimento de cuidados diários com a saúde, além do estímulo a interação social, condição que potencializa a independência, autoestima e autoconfiança, favorecendo o resgate dos valores pessoais, familiares e sociais, potencializando assim, a adoção de comportamentos saudáveis ${ }^{24}$.

Quanto às oficinas terapêuticas (OT), estas representam uma ferramenta importante no processo de ressocialização, inserção individual e coletiva, na medida em que possibilita o trabalho, o agir e o pensar coletivo ${ }^{25}$. Estas ações possibilitam aos idosos uma elevação na autoestima, um fortalecimento em relação à interação social, melhoria da resposta de estimulação psíquica, cognitiva e motora ${ }^{7}$.

Dentre as OT identificadas, destacam-se a de "jogos interativos", "jogo da memória" e "pintura e desenhos" conhecida na literatura como "Oficina de Arterapia", que foram voltadas para estimulação do raciocínio lógico, memória e interação dos idosos residentes nas ILPI's. Além de trazer consigo a ideia de que por trás de cada trabalho feito, existe um pro-

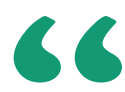

Os cuidados de enfermagem integram-se no uso do conjunto de ações que ao serem desenvolvidas permitem identificar as necessidades de saúde dos idosos, assim como
implementação
de atividades
educativas.

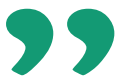

cesso que será construído com o tempo e isso trará um resgate do individual, da personalidade de cada pessoa ${ }^{26,27}$. Assim, essas oficinas auxiliam na recuperação do ser individual, agindo por meio da estimulação da psicomotricidade, melhorando inclusive sua integração social ${ }^{28}$.

No que diz respeito às práticas de atividades neuromotoras realizadas nas ILPI's, destacaram-se o boliche, a peteca, o bilboquê e alongamentos corporais. Esses exercícios exigem maior concentração, ampliam as possibilidades de movimento e quando realizados de forma lúdica, favorecem a descontração e a socialização, auxiliando os idosos a superar os desafios motores e cognitivos do cotidiano ${ }^{29}$. Baseando-se nos fatos citados, encontramos a importância da presença das atividades no dia a dia dos idosos. As atividades familiarizadas obtiveram maior adesão entre os idosos, proporcionaram além do estímulo neuromotor, o resgate de memória por meio de recordações.

Os cuidados de enfermagem integram-se no uso do conjunto de ações que ao serem desenvolvidas permitem identificar as necessidades de saúde dos idosos, assim como implementação de atividades educativas. Neste sentido as ações encontradas nas ILPI's foram: anamnese, exame físico e curativos. Ambos os cuidados de enfermagem realizados com os idosos institucionalizados obtiveram um resultado positivo contribuindo significativamente melhora da qualidade de vida.

A Política Nacional de Saúde da Pessoa Idosa, instituída pela Portaria $n^{\circ}$ 2.528, de outubro de 2006, estabelece que as práticas de cuidado direcionados aos idosos exigem uma abordagem global, interdisciplinar e multidimensional, levando em consideração a interação entre fatores físicos, psicológicos e sociais que influenciam sua saúde. Sendo assim as intervenções precisam ser realizadas e orientadas, visando à promoção da autonomia e independência da pessoa idosa, estimulando-a para o autocuidado ${ }^{7}$.

Com isso a enfermagem desenvolve um papel determinante na execução e cumprimento das leis direcionadas aos idosos, promovendo a inclusão social indiscriminada dos idosos, respeitando suas capacidades e limitações. Contudo, ainda há muito a conquistar nessa área de conhecimento. Quando o enfermeiro atua junto à pessoa idosa residente em uma ILPI, esse trabalhador tem condições de tornar esse cuidado mais humanizado, acolhedor, avaliativo, integral, podendo 
contribuir para melhoria da qualidade de vida do idoso institucionalizado ${ }^{30}$.

\section{CONCLUSÃO}

Com presente estudo, identificou-se que as os acadêmicos de enfermagem contribuem para a qualidade de vida de idosos institucionalizados em ILPI's. As ações realizadas colaboraram para a ma- nutenção da saúde dos idosos, de modo a proporcionar mudanças positivas no cotidiano. Os resultados obtidos levam-nos a refletir sobre questões referentes ao envelhecimento correlacionando idosos e qualidade de vida, e fatores que influenciam diretamente no novo processo de adaptação do idoso que está ou será institucionalizado, pois a institucionalização reflete diretamente no dia a dia dos idosos e seu modo de ser e agir em seu novo mundo.

Assim, a realização de atividades terapêuticas com os idosos, surtiram efeitos positivos na qualidade de vida dos idosos institucionalizados, os quais podem colaborar para a manutenção da saúde e interação social. E que, ao aplicar esses métodos, oferta-se uma forma de enveIhecimento saudável e ativo.

\section{Referências}

1. Miranda GMD, Mendes ACG, Silva ALA. 0 envelhecimento populacional brasileiro: desafios e consequências sociais atuais e futuras. Revista Brasileira de Geriatria e Gerontologia. 2019; 19(3); 507-519.

2. Brito FC, Litvoc $\mathrm{CJ}$. Envelhecimento: prevenção e promoção de saúde. $1^{\circ}$ ed. São Paulo: Atheneu; 2004.

3. Fechine BRA, Trompierl N. 0 processo de envelhecimento: as principais alterações que acontecem com o idoso com o passar dos anos. Revista Cientifica Internacional. 2012; 1(20).

4. UNITED NATIONS, Department of Economic and Social Affairs, Population Division [página na internet]. World Population Prospects: The 2012 Revision, Highlights and Advance Tables. [acesso em 16 de março de 2018]. Disponivel em: https://population.un.org/wpp/Publications/Files/WPP2012 HIGHLIGHTS.pdf

5. INSTITUTO BRASILEIRO DE GEOGRAFIA E ESTATÍSTICA [página na internet]. Número de idosos cresce $18 \%$ em 5 anos e ultrapassa 30 milhões em 2017 [acesso em 18 de março de 2020]. Disponível em: https:// agenciadenoticias.ibge.gov.br/agencia-noticias/2012-agencia-de-noticias/ noticias/20980-numero-de-idosos-cresce-18-em-5-anos-e-ultrapassa-30milhoes-em-2017

6. WORLD HEALTH ORGANIZATION [página na internet]. Envelhecimento ativo: uma política de saúde / World Health Organization; tradução Suzana Gontijo. - Brasília: Organização Pan-Americana da Saúde de [acesso em 18 de março de 2020]. Disponível em: http://bvsms.saude.gov.br/bvs/publicacoes/envelhecimento_ativo.pdf.

7. BRASIL. Ministério da Saúde. Envelhecimento e saúde da pessoa idosa: Cadernos de Atenção Básica 19 [internet]. Brasília; 2006. [Acesso em: 11 de junho de 2020]. Disponível em: http://bvsms.saude.gov.br/bvs/publicacoes/ evelhecimento_saude_pessoa_idosa.pdf.

8. Camargos MCS, Gonzaga MR, Costa JV, Bomfim WC. Estimativas de expectativa de vida livre de incapacidade funcional para Brasil e Grandes Regiões, 1998 e 2013. Ciência \& saúde coletiva. 2019; 24(3); 737-747.

9. Camargos MCS, Gonzaga MR. Viver mais e melhor? Estimativas de expectativa de vida saudável para a população brasileira. Caderno Saúde Pública. 2015; 31(7); 1460-1472.

10. Lima APM, Gomes KVL, Frota NM, Pereira FGF. Qualidade de vida sob a óptica da pessoa idosa institucionalizada. Revista Brasileira em Promoção da Saúde. 2016; 29(1); 14-19.

11. Dawali NW, Anacleto GMC, Witter C, Goulart RMM, Aquin RC. Envelhecimento e qualidade de vida: análise da produção científica da SciELo. Estudos de Psicologia. 2013; 30(3); 393-403.

12. Toldrá RC, Cordone RG, Arruda BA, Souto ACF. Promoção da saúde e da qualidade de vida com idosos por meio de práticas corporais. 0 Mundo da Saúde. 2014; 38(2); 159-168.

13. Born T, Abreu CMG. 0 cuidado ao idoso em instituição de longa permanência. Revista Gerontologia. 2008; 4; 7-14.

14. Gonçalves AL. A psicomotricidade aplicada à terceira idade. Rio de Janeiro: UCAM Universidade Cândido Mendes, 2011. Monografia de Pós-Gradu- ação em Psicomotricidade.

15. Silva AFL, Ribeiro CDM, Silva JAG. Thinking of university extension as a health education field: an experience at the Fluminense Federal University, Brazil. Interface-comunicação [revista em Internet]. 2013; acesso 13 de junho de 2020; 17 . Disponível em: http:// www.redalyc.org/html/1801/ $180127931010 /$

16. Silva W, Lucena ALR, Araújo MJA, Januário DC, Viera KFLI, Costa VRRAB. Ações educativas vivenciadas com idosos: um relato de experiência. Revista de Ciências da Saúde Nova Esperança. 2017; 15(3):31-36.

17. Freire P. Extensão ou Comunicação. 13a ed. São Paulo: Paz e Terra; 2006. 18. Lucena ALR, Freitas FFQ, Vieira KFL, Matos SDO. Ensinando e aprendendo com idosos: relato de experiência. Journal of Research Fundamental Care. 2016; 8(2):4131-4141.

19. Ferreira OGL, Maciel SC, Costa SMG, Silva AO, Moreira MASP. Envelhecimento ativo e sua relação com a independência funcional. Texto \& contexto Enfermagem. 2012; 21(3):513-518.

20. Botelho L, Cunha C, Macedo M. 0 método da revisão integrativa nos estudos organizacionais. Revista Gestão e Sociedade. 2013; 5(11):121-136. 21. Leite MT, Hildebrandt LM, Kirchner RM, Winck MT, Silva LAA, Franco GP. Estado cognitivo e condições de saúde de idosos que participam de grupos de convivência. Revista Gaúcha de Enfermagem. 2012; 33(4):64-71.

22. Santana JO, Tavares ML, Pereira ET. Lazer e saúde: Programa de atividade física e de lazer melhora capacidade funcional de idosos institucionalizados. Motricidade. 2012; 8:725-733.

23. Mallmann DG, Galindo Neto NM, Sousa JC, Vasconcelos EMR. Educação em saúde como principal alternativa para promover a saúde do idoso. Ciência \& Saúde Coletiva. 2015; 20(6): 1763-1772.

24. Souza AMV, Abreu AM, Souza AG, Pereira KG, Souza LPS, Figueiredo MFS et al. Grupos educativos para idosos na Estratégia Saúde da Família: uma nova perspectiva. Revista Norte Mineira de Enfermagem. 2014; 3(2):162-169 25. Ibiapina ARS, Monteiro CFS, Alencar DC, Fernandes MA, Costa Filho AAI. Oficinas Terapêuticas e as mudanças sociais em portadores de transtorno mental. Escola Anna Nery. 2017; 21(3).

26. Parola V, Coelho A, Neves H, Almeida M, Gil I, Mouro A. et al . Efectos de un programa de estimulación cognitiva en ancianos institucionalizados. Revista de Enfermagem de Referência. 2019; 1(20): 47-56.

27. Aguiar AP, Marci R. Promovendo a Qualidade de Vida dos Idosos Através da Arteterapia. Revista de Pesquisa: Cuidado é fundamental. 2010; 1(2): 710-7813.

28. Carvalho NC. Dinâmicas para Idosos. Petrópolis-RJ: Ed. Vozes; 2009. 29. Souza, CH. Exercícios para o desenvolvimento das capacidades neuromotoras para idosos. Londrina: Centro de Pesquisa em Ciências da Saúde. Universidade Norte do Paraná, 2015. Relatório técnico de mestrado profissional em exercício físico na promoção da saúde.

30. Rodrigues RAP, Kusumota L, Marques S, Fabrício SCC , Cruz IR , Lange C. Política Nacional de Atenção ao Idoso e a Contribuição da Enfermagem. Texto Contexto Enfermagem. 2007; 16(3): 536-545. 